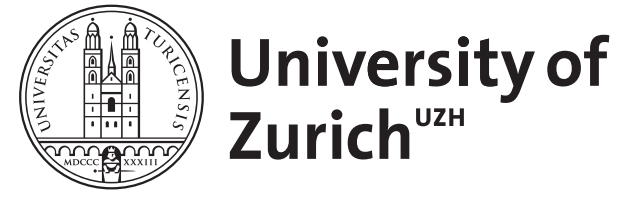
Archive

University of Zurich

University Library

Strickhofstrasse 39

CH-8057 Zurich

www.zora.uzh.ch

Year: 2006

\title{
Evolutionary Stable Stock Markets
}

Evstigneev, Igor V ; Hens, Thorsten ; Schenk-Hoppe, Klaus Reiner

DOI: https://doi.org/10.1007/s00199-005-0607-8

Posted at the Zurich Open Repository and Archive, University of Zurich

ZORA URL: https://doi.org/10.5167/uzh-95113

Journal Article

Published Version

Originally published at:

Evstigneev, Igor V; Hens, Thorsten; Schenk-Hoppe, Klaus Reiner (2006). Evolutionary Stable Stock Markets. Economic Theory, 27:449-468.

DOI: https://doi.org/10.1007/s00199-005-0607-8 


\title{
Evolutionary stable stock markets ${ }^{\star}$
}

\author{
Igor V. Evstigneev ${ }^{1}$, Thorsten Hens ${ }^{2}$, and Klaus Reiner Schenk-Hoppé $e^{2,3}$ \\ 1 School of Economic Studies, University of Manchester, Oxford Road, \\ Manchester M13 9PL, UNITED KINGDOM (e-mail: igor.evstigneev@man.ac.uk) \\ 2 Institute for Empirical Research in Economics, University of Zurich, \\ Blümlisalpstrasse 10, 8006 Zürich, SWITZERLAND (e-mail: thens@iew.unizh.ch) \\ 3 Leeds University Business School and School of Mathematics, University of Leeds, \\ Leeds LS2 9JT, UNITED KINGDOM (e-mail: k.r.schenk-hoppe@ leeds.ac.uk)
}

Received: October 7, 2003; revised version: January 18, 2005

Summary. This paper shows that a stock market is evolutionary stable if and only if stocks are evaluated by expected relative dividends. Any other market can be invaded in the sense that there is a portfolio rule that, when introduced on the market with arbitrarily small initial wealth, increases its market share at the incumbent's expense. This mutant portfolio rule changes the asset valuation in the course of time. The stochastic wealth dynamics in our evolutionary stock market model is formulated as a random dynamical system. Applying this theory, necessary and sufficient conditions are derived for the evolutionary stability of portfolio rules when relative dividend payoffs follow a stationary Markov process. These local stability conditions lead to a unique evolutionary stable portfolio rule according to which assets are evaluated by expected relative dividends (with respect to the objective probabilities).

Keywords and Phrases: Evolutionary finance, Portfolio theory, Incomplete markets.

JEL Classification Numbers: G11, D52, D81.

\footnotetext{
* We are grateful to Jarrod Wilcox and William Ziemba for valuable comments. Financial support by the national center of competence in research "Financial Valuation and Risk Management" is gratefully acknowledged. The national centers in research are managed by the Swiss National Science Foundation on behalf of the federal authorities.
}

Correspondence to: K.R. Schenk-Hoppé 


\section{Introduction}

The expected discounted dividends model is one of the cornerstones of finance. According to this model the rational and fair value of common stocks is given by the expected value of the discounted sum of future dividends paid out by the company. Indeed in the very long run the trend of stock market prices coincides with the trend of the dividends paid by the companies. Yet over shorter horizons (sometimes even for decades) stock market prices can considerably deviate from their fundamentals. This phenomenon, called excess volatility, was first pointed out by Shiller (1981). While models based on complete rationality have difficulties to cope with excess volatility, models based on adaptive behavior typically go to the other extreme and generate too irregular price dynamics. We suggest here a solution in between these two extremes.

This paper considers a stock market model with a heterogenous population of portfolio rules. In our model rationality is important on the level of the market since market selection may ultimately give pressure for selecting the rational portfolio rules. It turns out that only a rational market in which assets are evaluated by expected relative dividends is evolutionary stable. Any other market can be invaded, i.e. there are portfolio rules that will gain market wealth, and hence the valuation of assets changes. While, as in De Long et al. (1990), rational strategies clearly face the risk that there are too many irrational strategies, any set of irrational strategies is however more easily turned over by invasion of even a small fraction of slightly different strategies. That is to say, every now and then the market can be displaced from its rational valuation by a big push of irrationality but eventually the market selection pressure will lead the market back to the rational valuation because from any irrational market there exists a sequence of small and nearby innovations leading back to the rational market. This stability property may be the explanation that on long-term averages stock markets look quite rational while severe departures are possible in the short- and medium-term.

In a sense our results give support to a long-held belief by Friedman (1953), Fama (1965) and others, who argued that the market naturally selects for rational strategies which, in effect, would lead to market efficiency. However, our paper also makes clear that the mutation force has to be added to the selection argument in order to prove this conjecture. Considering only the market selection process, the economy can get stuck at any situation in which all investors use the same portfolio rule. Moreover, our paper shows which portfolio rules can successfully enter which market. For example, an irrational market can be turned over by portfolio rules that are not themselves rational portfolio rules. The rational portfolio rule actually may fail to invade an irrational market.

To make these ideas precise, we study an asset market (complete or incomplete) where a finite number of portfolio rules manage capital by iteratively reinvesting in a fixed set of long-lived assets. In every period assets pay dividends according to the realization of a stationary Markov process in discrete time. In addition to the exogenous wealth increase due to dividends, portfolio rules face endogenously determined capital gains or losses. Portfolio rules are encoded as non-negative vectors of expenditure shares for assets. The set of portfolio rules considered is 
not restricted to those generated by expected utility maximization. It may as well include investment rules favored by behavioral finance models. Indeed any portfolio rule that is adapted to the information filtration is allowed in our framework.

Portfolio rules compete for market capital that is given by the total value of all assets in every period in time. The endogenous price process provides a market selection mechanism along which some portfolio rules gain market capital while others lose. We give a description of the market selection process from a random dynamical systems perspective. In each period in time the evolution of the distribution of market capital, i.e. the wealth shares of the portfolio rules as percentages of total market wealth, is given by a map that depends on the exogenous process determining the asset payoffs. An equilibrium in this model is provided by a distribution of wealth shares across portfolio rules that is invariant under the market selection process. Provided there are no redundant assets, every invariant distribution of market shares is generated by a monomorphic population, i.e. all investors with strictly positive wealth use the same portfolio rule at such an equilibrium. These invariant distributions are fixed points in an appropriate space.

A portfolio rule is evolutionary stable if the state in which this rule has total market wealth is robust against the entry of new portfolio rules with sufficiently small wealth. In other words, an evolutionary stable portfolio rule drives out any mutation. Criteria for evolutionary stability as well as evolutionary instability are derived for such fixed points. The derivation is via the linearization of the local dynamics. These sufficient and necessary conditions can be used to single out one particular portfolio rule, denoted by $\lambda^{*}$, that is the unique evolutionary stable portfolio rule. The rule $\lambda^{*}$ is the only one that has highest exponential growth rate at its own market prices. In a sense, when the population pursues the evolutionary stable portfolio rule, it plays the "best response against itself." Moreover, any other market with one portfolio rule can successfully be invaded by a slightly different rule, i.e. the market can be destabilized by mutant portfolio rules that are small variations of the incumbent portfolio rule. This mutant portfolio rule will then lead to a change of the asset valuation in the market.

An explicit formula for the $\lambda^{*}$-rule is given, and it is applicable to real financial markets. This $\lambda^{*}$-rule prescribes to divide one's wealth proportionally to the expected relative dividends of assets. It is therefore justified to call a financial market with $\lambda^{*}$ only rational, while any other market is termed irrational.

The effect of this $\lambda^{*}$-rule on asset prices is to equalize all assets' expected relative returns - in particular asset pricing is log-optimal in the sense of Luenberger (1997, Chapter 15). It is well known that log-optimal pricing is obtained if all investors have logarithmic von Neumann-Morgenstern utilities (Kraus and Litzenberger, 1975). The portfolio rule $\lambda^{*}$ could therefore be obtained as well as in an idealized market with a single representative agent having rational expectations. For a market selection model based on rational expectations see Blume and Easley (2000) and Sandroni (2000). Our paper shows that such an idealized market with rational expectations can be justified by evolutionary reasoning.

A further implication of our evolutionary stability result is that, among all proportional portfolio rules, only the $\lambda^{*}$-rule is a candidate for a globally evolutionary stable portfolio rule, i.e. convergence to the status quo when the disturbance of the 
market can be large in the sense that any initial distribution of wealth is permitted. Indeed, global stability of the $\lambda^{*}$-rule has recently been proved for the case of short-lived assets Evstiigneev et al. (2002). Simulations with simple (i.e. deterministic) portfolio rules also indicate that the $\lambda^{*}$-rule has this property in the case of long-lived assets Hens et al. (2002). An analytical proof of this finding is still warranted.

Our approach is related to the classical finance approach to maximize the expected logarithm of the growth rate of relative wealth for some exogenously given return process. From this perspective we show which portfolio rule maximizes the expected logarithm of the growth rate of wealth in a model with endogenously determined returns. Following the early work by Kelly (1956) and Breiman (1961), in a series of papers Hakansson (1970), Thorp (1971), Algoet and Cover (1988), and Karatzas and Shreves (1998), among others, have explored this maximum growth perspective. Computing the maximum growth portfolio is a stochastic non-linear programming problem. Even if one restricts attention to i.i.d. returns, when markets are incomplete and there are more than two assets, there is no explicit solution to this investment problem in general. To overcome this problem, numerical algorithms to compute the maximum growth portfolio have been provided by Algoet and Cover (1988) and Cover $(1984,1991)$. Our result is interesting also in this respect because the simple portfolio rule that we obtain shows that considering the equilibrium consequences of this maximization does not make matters more complicated but rather much easier. Indeed, as mentioned above, the portfolio rule $\lambda^{*}$ can be characterized as the unique portfolio rule that maximizes the logarithm of the growth rate of relative wealth in a population in which the rule itself determines the returns. Note however that applying the $\lambda^{*}$-rule does not require the solution of any optimization problem. The forces of market selection and its robustness against mutations make the $\lambda^{*}$-rule look such a smart investment rule.

The next section presents the financial market model which has the mathematical structure of a random dynamical system. The model is based on Lucas (1978)'s infinite horizon asset market model with long-lived assets and a single perishable consumption good. This model is populated by a finite number of different portfolio rules that are adapted to the information filtration. The evolution of the stochastic wealth distribution is governed by a sequence of short-run equilibria. In Section 3 we define the long-run equilibrium concepts and different stability notions. In particular we define invariant distributions of relative wealth and show that deterministic ones are characterized by monomorphic populations, i.e. where all investors use the same portfolio rule. We then define evolutionary stability of invariant distributions as robustness to the entry of other portfolio rules. Section 4 contains the main result. Section 5 concludes.

\section{An evolutionary stock market model}

This section introduces an infinite horizon asset market model with long-lived assets and a single perishable consumption good, as in the seminal paper Lucas (1978).

There are $K \geq 1$ long-lived assets and cash. Time is discrete and denoted by $t=0,1, \ldots$. Each asset $k=1, \ldots, K$ pays off a dividend per share at the 
beginning of every period and before trade takes place in this period. $D_{t}^{k} \geq 0$ denotes the total dividend paid to all shareholders of asset $k$ at the beginning of period $t$. Assume that $\sum_{k} D_{t}^{k}>0 .{ }^{1} D_{t}^{k}$ depends on the history of states of the world $\omega^{t}=\left(\ldots, \omega_{0}, \ldots, \omega_{t}\right)$ where $\omega_{t} \in S$ is the state revealed at the beginning of period $t$. For technical convenience (and without loss of generality) we assume infinite histories. $S$ is assumed to be finite, and every state is drawn with some strictly positive probability.

Dividend payoffs are in cash. Cash is only used to buy consumption goods-in particular it cannot be used to store value. Assets are issued at time 0 . The initial supply of every asset $k, s_{0}^{k}$, is normalized to 1 . At any period in time the supply remains constant: $s_{t}^{k}=s_{0}^{k}$. The supply of cash $s_{t}^{0}$ is given by the total dividends of all assets.

There are finitely many portfolio rules indexed by $i=1, \ldots, I, I \geq 2$, each is pursued by a group of investors. Each investor's claim is equal to his initial share, and consumption rates are identical within groups. The portfolio rule $i$ is a time- and history-dependent vector of proportions, denoted by $\lambda_{t}^{i}\left(\omega^{t}\right)=\left(\lambda_{t, k}^{i}\left(\omega^{t}\right)\right)_{k=0, \ldots, K}$ with $0 \leq \lambda_{t, k}^{i}\left(\omega^{t}\right) \leq 1$ for all $k$ and $\sum_{k=0}^{K} \lambda_{t, k}^{i}\left(\omega^{t}\right)=1$. For each $k \geq 1, \lambda_{t, k}^{i}\left(\omega^{t}\right)$ is the fraction of the wealth portfolio rule $i$ assigns to the purchase of the risky asset $k$ in period $t$, while $\lambda_{t, 0}^{i}\left(\omega^{t}\right)$ is the fraction of wealth held in cash.

In the following discussion we assume that everything is well-defined. In particular prices are assumed to be strictly positive. Sufficient conditions ensuring well-definedness are provided after the full derivation of the model.

Portfolio rule $\lambda_{t}^{i}\left(\omega^{t}\right)$ with wealth $w_{t}^{i}$ purchases at the beginning of period $t$ the portfolio

$$
\theta_{t, k}^{i}=\frac{\lambda_{t, k}^{i}\left(\omega^{t}\right) w_{t}^{i}}{p_{t}^{k}} \quad k=0,1, \ldots, K .
$$

$\theta_{t, 0}^{i}$ is the units of cash and $\theta_{t, k}^{i}$ is the units of assets held. Since we have normalized the supply of the long-lived assets to $1, \theta_{t, k}^{i}$ is the percentage of all shares issued of asset $k$ that portfolio rule $i$ purchases. $p_{t}^{k}$ denotes the market clearing price of asset $k$ in period $t$. We normalize the price for cash $p_{t}^{0}=1$ in every period $t$. The price of the consumption good is also the numeraire.

For any portfolio holdings $\left(\theta_{t}^{i}\right)_{i=1, \ldots, I}$, the market equilibrium conditions for cash and long-lived assets are

$$
\sum_{i=1}^{I} \theta_{t, k}^{i}=s_{t}^{k}, \quad k=0, \ldots, K,
$$

where the supply of the risky assets is $s_{t}^{k}=1$, while the supply of cash is

$$
s_{t}^{0}=\sum_{k=1}^{K} D_{t}^{k}\left(\omega^{t}\right)>0
$$

with strict positivity by the assumption that at least one asset pays a dividend.

\footnotetext{
1 This assumption avoids "dead" periods in which no dividends are paid.
} 
The budget constraint of portfolio rule $i$ in every period $t=0,1, \ldots$

$$
\sum_{k=0}^{K} p_{t}^{k} \theta_{t, k}^{i}=w_{t}^{i}
$$

is fulfilled because the fractions $\lambda_{t, k}^{i}\left(\omega^{t}\right), k=0, \ldots, K$, sum to one, see (1).

Since the consumption good is perishable, the wealth of portfolio rule $i$ (in terms of the price of the consumption good) at the beginning of period $t+1$ and after dividends are payed is

$$
w_{t+1}^{i}=\sum_{k=1}^{K}\left(D_{t+1}^{k}\left(\omega^{t+1}\right)+p_{t+1}^{k}\right) \theta_{t, k}^{i} .
$$

Wealth can change over time because of dividend payments and capital gains. Since the cash $\theta_{t, 0}^{i}$ held by every portfolio rule is consumed, the amount of cash available in any one period stems only from the current's period dividend payments.

The market-clearing price $p_{t}^{k}$ for the risky assets $(k \geq 1)$ can be derived from (2) by inserting (1). One finds

$$
p_{t}^{k}=\sum_{i=1}^{I} \lambda_{t, k}^{i}\left(\omega^{t}\right) w_{t}^{i}=\lambda_{t, k}\left(\omega^{t}\right) w_{t}
$$

where $\lambda_{t, k}=\left(\lambda_{t, k}^{1}, \ldots, \lambda_{t, k}^{I}\right)$ and $w_{t}^{T}=\left(w_{t}^{1}, \ldots, w_{t}^{I}\right)$. Thus the price of asset $k$ is the weighted sum of the portfolio rules.

Inserting (1) and (6) in (5) yields

$$
w_{t+1}^{i}=\sum_{k=1}^{K}\left(D_{t+1}^{k}\left(\omega^{t+1}\right)+\lambda_{t+1, k}\left(\omega^{t+1}\right) w_{t+1}\right) \frac{\lambda_{t, k}^{i}\left(\omega^{t}\right) w_{t}^{i}}{\lambda_{t, k}\left(\omega^{t}\right) w_{t}}
$$

This is an implicit equation for portfolio rule $i$ 's wealth in period $t+1, w_{t+1}^{i}$, for a given distribution of wealth $w_{t}$ across portfolio rules in period $t$. Define

$$
A_{t}^{i}=\sum_{k=1}^{K} D_{t+1}^{k}\left(\omega^{t+1}\right) \theta_{t, k}^{i}, \quad \text { where } \quad \theta_{t, k}^{i}=\frac{\lambda_{t, k}^{i}\left(\omega^{t}\right) w_{t}^{i}}{\lambda_{t, k}\left(\omega^{t}\right) w_{t}}
$$

The time index refers to the dependence on wealth. The dividend income of portfolio rule $i, A_{t}^{i}$ and the portfolio $\theta_{t, k}^{i}$ both depend on the wealth in period $t$. (7) can now be written as

$$
w_{t+1}^{i}=A_{t}^{i}+\sum_{k=1}^{K} \theta_{t, k}^{i} \lambda_{t+1, k}\left(\omega^{t+1}\right) w_{t+1}
$$

and thus

$$
w_{t+1}=A_{t}+\Theta_{t} \Lambda_{t+1}\left(\omega^{t+1}\right) w_{t+1}
$$

where $\Lambda_{t+1}\left(\omega^{t+1}\right)^{T}=\left(\lambda_{t+1,1}\left(\omega^{t+1}\right)^{T}, \ldots, \lambda_{t+1, K}\left(\omega^{t+1}\right)^{T}\right) \in \mathbb{R}^{I \times K}$ is the matrix of portfolio rules, and $\Theta_{t} \in \mathbb{R}^{I \times K}$ is the matrix of portfolios in period $t$. 
$A_{t}^{T}=\left(A_{t}^{1}, \ldots, A_{t}^{I}\right) \in \mathbb{R}^{I}$ are the dividends payments, and $\Theta_{t} \Lambda_{t+1}\left(\omega^{t+1}\right) w_{t+1}$ are the capital gains.

Solving the linear equation (9) gives an explicit law of motion governing the evolution of the wealth distribution over portfolio rules. One has

$$
w_{t+1}=\left[\operatorname{Id}-\Theta_{t} \Lambda_{t+1}\left(\omega^{t+1}\right)\right]^{-1} A_{t}
$$

(assuming existence of the inverse matrix) with Id being the identity matrix in $\mathbb{R}^{I \times I}$. The following result ensures that the evolution of wealth (11) is mathematically well-defined.

The next two assumptions are imposed throughout the text.

(A.1) Consumption takes place but does not exhaust any portfolio rule's wealth, i.e. $0<\lambda_{t, 0}^{i}\left(\omega^{t}\right)<1$ for all $i$, $t$ and $\omega^{t}$.

(A.2) There is at least one completely diversified portfolio rule, i.e. there is a j such that $\lambda_{t, k}^{j}\left(\omega^{t}\right)>0$ for all $k=1, \ldots, K, t$ and $\omega^{t}$.

Proposition 1. Suppose $w_{0}>0,(A .1)$ holds, and (A.2) is satisfied for some portfolio rule with $w_{0}^{j}>0$. Then the evolution of wealth (11) is well-defined in all periods in time. Moreover, for every $i=1, \ldots, I, w_{t}^{i}>0$ if and only if $w_{0}^{i}>0$.

Proof of Proposition 1. It suffices to prove the following which ensures that every transition from time $t$ to $t+1$ is well-defined: Suppose $w_{t}>0$, (A.1) holds, and (A.2) is satisfied for some portfolio rule with $w_{t}^{j}>0$. Then (11) is well-defined, $w_{t+1}>0$, and, moreover, $w_{t+1}^{i}>0$ if and only if $w_{t}^{i}>0$ for every $i=1, \ldots, I$. In particular a portfolio rule $j$ that satisfies (A.2) and has strictly positive initial wealth $w_{0}^{j}>0$ fulfills $w_{t}^{j}>0$ for all $t$.

We show first that the matrix $C:=\mathrm{Id}-\Theta_{t} \Lambda_{t+1}\left(\omega^{t+1}\right)$ is invertible by proving that it has a column dominant diagonal Murata (1977, Corollary p. 22). $C$ has entries

$$
C_{j j}=1-\sum_{k=1}^{K} \bar{\lambda}_{k}^{j} \frac{\lambda_{k}^{j} w^{j}}{\lambda_{k} w} \quad \text { and } \quad C_{i j}=-\sum_{k=1}^{K} \bar{\lambda}_{k}^{j} \frac{\lambda_{k}^{i} w^{i}}{\lambda_{k} w} \quad(i \neq j)
$$

on the diagonal and off-diagonal, respectively, where $\bar{\lambda}_{k}^{i}=\lambda_{t+1, k}^{i}\left(\omega^{t+1}\right), \lambda_{k}^{i}=$ $\lambda_{t, k}^{i}\left(\omega^{t}\right)$, and $w=w_{t}$ for notational ease. All entries are well-defined because prices $\lambda_{k} w \geq \lambda_{k}^{j} w^{j}>0$ (for some $j$ ) by our assumption.

The condition for a column dominant diagonal is in particular satisfied, if for every $j=1, \ldots, I$,

$$
\left|C_{j j}\right|>\sum_{i \neq j}\left|C_{i j}\right|
$$

Off-diagonal entries are non-positive, i.e. $C_{i j} \leq 0$ for $i \neq j$. The diagonal elements are strictly positive, i.e. $C_{j j}>0$, since $0 \leq \lambda_{k}^{j} w^{j} /\left(\lambda_{k} w\right) \leq 1$ and therefore

$$
C_{j j} \geq 1-\sum_{k=1}^{K} \bar{\lambda}_{k}^{j}=1-\left(1-\bar{\lambda}_{0}^{j}\right)=\bar{\lambda}_{0}^{j}>0
$$


according to assumption (A.1).

Thus (12) is equivalent to

$$
1>\sum_{i=1}^{I} \sum_{k=1}^{K} \bar{\lambda}_{k}^{j} \frac{\lambda_{k}^{i} w^{i}}{\lambda_{k} w}
$$

Since the right-hand side of the last equation is

$$
\sum_{k=1}^{K} \bar{\lambda}_{k}^{j} \sum_{i=1}^{I} \frac{\lambda_{k}^{i} w^{i}}{\lambda_{k} w}=\sum_{k=1}^{K} \bar{\lambda}_{k}^{j}=1-\bar{\lambda}_{0}^{j}
$$

and $\bar{\lambda}_{0}^{j}>0$ by assumption, (13) holds true. Thus $C$ is invertible.

The matrix $C$ has strictly positive diagonal entries and non-positive off-diagonal entries. Thus, Murata (1977, Theorem 23, p. 24) ensures that $w_{t+1} \geq 0$ if $A_{t} \geq$ 0 (see (8) for the definition of $A_{t}$ ). Clearly, $A_{t} \geq 0$ if $w_{t} \geq 0$. This implies $\lambda_{t+1, k}\left(\omega^{t+1}\right) w_{t+1} \geq 0$ for all $k$.

Our assumptions ensure that there is a portfolio rule $j$ which is completely diversified and has strictly positive wealth. This implies $\theta_{t, k}^{j}>0$ for all $k$. Because at least one asset pays a strictly positive dividend, $A_{t}^{j}>0$. Equation (7) implies, together with the above result that prices in period $t+1$ are non-negative, $w_{t+1}^{j}>0$.

By assumption (A.2) one also has $\lambda_{t+1, k}^{j}\left(\omega^{t+1}\right)>0$ for all $k$. Since for each investor with $w_{t}^{i}>0, \theta_{t, k}^{i}>0$ for some $k$. (7) further implies that $w_{t+1}^{i}>0$ for every investor with $w_{t}^{i}>0$. Obviously, $w_{t+1}^{i}=0$ if $w_{t}^{i}=0$. This completes the proof.

Proposition 1 ensures that the evolution of the wealth distribution on $\mathbb{R}_{+}^{I}$ is welldefined: for given $w_{t},(11)$ yields the distribution of wealth $w_{t+1}$ in the subsequent period in time. We can state the law of motion in the convenient form ${ }^{2}$

$$
w_{t+1}=f_{t}\left(\omega^{t+1}, w_{t}\right)
$$

where

$$
\begin{aligned}
& f_{t}\left(\omega^{t+1}, w_{t}\right)= \\
& \quad\left[\operatorname{Id}-\left[\frac{\lambda_{t, k}^{i}\left(\omega^{t}\right) w_{t}^{i}}{\lambda_{t, k}\left(\omega^{t}\right) w_{t}}\right]_{i, k} \Lambda_{t+1}\left(\omega^{t+1}\right)\right]^{-1}\left[\sum_{k=1}^{K} D_{t+1}^{k}\left(\omega^{t+1}\right) \frac{\lambda_{t, k}^{i}\left(\omega^{t}\right) w_{t}^{i}}{\lambda_{t, k}\left(\omega^{t}\right) w_{t}}\right]_{i}
\end{aligned}
$$

The final step is to derive the law of motion for the portfolio rules' market shares. This will complete the derivation of the evolutionary stock market model.

The following assumption is imposed throughout the remainder of this paper.

2 It is also convenient to define $w_{t+1}=(0, \ldots, 0)$, if $w_{t}=(0, \ldots, 0)$. 
(B.1) There is a common constant consumption rate, i.e. $\lambda_{t, 0}^{i}\left(\omega^{t}\right)=\lambda_{0}$.

It is clear that, other things being equal, a smaller rate of consumption leads to a higher growth rate of wealth. Without assumption (B.1) the evolution of wealth would be biased in favor of portfolio rules with higher saving rate. Since we want to analyze the relative performance of different asset allocation rules no rule should have an disadvantage in terms of the rate at which wealth is withdrawn from it.

Aggregating (7) over portfolio rules, one finds

$$
\begin{aligned}
W_{t+1} & =\sum_{k=1}^{K} D_{t+1}^{k}\left(\omega^{t+1}\right)+\sum_{k=1}^{K} \lambda_{t+1, k}\left(\omega^{t+1}\right) w_{t+1} \\
& =D_{t+1}\left(\omega^{t+1}\right)+\left(1-\lambda_{0}\right) W_{t+1}
\end{aligned}
$$

where $D_{t+1}\left(\omega^{t+1}\right)=\sum_{k=1}^{K} D_{t+1}^{k}\left(\omega^{t+1}\right)$ is the aggregate dividend payment. The last equality holds because $\sum_{k=1}^{K} \lambda_{t+1, k} w_{t+1}=\sum_{i=1}^{I} \sum_{k=1}^{K} \lambda_{t+1, k}^{i} w_{t+1}^{i}=(1-$ $\left.\lambda_{0}\right) \sum_{i=1}^{I} w_{t+1}^{i}$.

Equation (15) implies

$$
W_{t+1}=\frac{D_{t+1}\left(\omega^{t+1}\right)}{\lambda_{0}}
$$

The economy grows (or declines) with rate $D_{t+1}\left(\omega^{t+1}\right) / \lambda_{0} W_{t}$. The growth rate is thus the ratio of the rate at which additional wealth is injected by dividends, $D_{t+1}\left(\omega^{t+1}\right) / W_{t}$, to the rate at which wealth is withdrawn from the process for consumption, $\lambda_{0}$.

The market share of investor $i$ is $r_{t}^{i}=w_{t}^{i} / W_{t}$. Using (16) and exploiting the particular structure of the variables (8) that define the law of motion (14), we obtain

$$
r_{t+1}=\frac{\lambda_{0}}{D_{t+1}\left(\omega^{t+1}\right)} f_{t}\left(\omega^{t+1}, r_{t}\right)
$$

or, equivalently,

$$
\begin{aligned}
& r_{t+1}= \\
& \lambda_{0}\left(\operatorname{Id}-\left[\frac{\lambda_{t, k}^{i}\left(\omega^{t}\right) r_{t}^{i}}{\lambda_{t, k}\left(\omega^{t}\right) r_{t}}\right]_{i, k} \Lambda_{t+1}\left(\omega^{t+1}\right)\right)^{-1}\left[\sum_{k=1}^{K} d_{t+1}^{k}\left(\omega^{t+1}\right) \frac{\lambda_{t, k}^{i}\left(\omega^{t}\right) r_{t}^{i}}{\lambda_{t, k}\left(\omega^{t}\right) r_{t}}\right]_{i}
\end{aligned}
$$

where

$$
d_{t+1}^{k}\left(\omega^{t+1}\right)=\frac{D_{t+1}^{k}\left(\omega^{t+1}\right)}{D_{t+1}\left(\omega^{t+1}\right)}
$$

is the relative dividend payment of asset $k$. Equation (17) is referred to as the market selection process.

The wealth of an investor $i$ in any period in time can be derived from their market share and the aggregate wealth, defined by (16), as

$$
w_{t+1}^{i}=\frac{D_{t+1}\left(\omega^{t+1}\right)}{\lambda_{0}} r_{t+1}^{i}
$$




\section{Evolutionary stability}

This section introduces the stability concepts needed to formalize the notion of evolutionary stability of portfolio rules. Stability is to be understood as properties of the long-run behavior of the portfolio rules' wealth shares under the market selection process. Our analysis is restricted to the stationary case which is achieved by assuming that the calender date neither enters through portfolio rules nor the dividend process, i.e. the model becomes stationary; only the observed history matters.

(B.2) Portfolio rules are stationary, i.e. $\lambda_{t, k}^{i}\left(\omega^{t}\right)=\lambda_{k}^{i}\left(\omega^{t}\right)$ for all $i=1, \ldots, I$ and $k=1, \ldots, K$.

(B.3) Relative dividend payments are stationary and depend only on the current state of nature, i.e. $d_{t}^{k}\left(\omega^{t}\right)=d^{k}\left(\omega_{t}\right)$ for all $k=1, \ldots, K$.

Assumption (B.3) is fulfilled, for instance, if $D_{t+1}^{k}\left(\omega^{t+1}\right)=d^{k}\left(\omega_{t+1}\right) W_{t}$ with $W_{t}=\sum_{i} w_{t}^{i}$, i.e. the dividend payment of every asset has an idiosyncratic component $d^{k}\left(\omega_{t+1}\right)$ (depending only on the state of nature in the respective period) and an aggregate component $W_{t}$. Dividends grow or decline with the same rate as aggregate wealth.

Under these assumptions, the market selection process (17) generates a random dynamical system (Arnold 1998) on the simplex $\Delta^{I}=\left\{r \in \mathbb{R}^{I} \mid r^{i} \geq 0, \sum_{i} r^{i}=1\right\}$. For any initial distribution of wealth $w_{0} \in \mathbb{R}_{+}^{I}$, (17) defines the path of market shares on the event tree with branches $\omega^{t}$. The initial distribution of market shares is $\left(r_{0}^{i}\right)_{i}=\left(w_{0}^{i} / W_{0}\right)_{i}$. Formally, this can be stated as follows.

Denote by $\Omega=S^{\mathbb{Z}}$ the set of all sequences of states of nature $\omega=\left(\omega_{t}\right)_{t \in \mathbb{Z}}$. Denote the right-hand side of (17) by $h\left(\omega^{t+1}, r_{t}\right): \Delta^{I} \rightarrow \Delta^{I}$. This map is independent of the calender date by assumptions (B.1)-(B.3). Define $\varphi(t, \omega, r)=$ $h\left(\omega^{t+1}, \cdot\right) \circ \ldots \circ h\left(\omega^{1}, r\right)$ for all $t \geq 1$, and $\varphi(0, \omega, r)=r . \varphi(t, \omega, r)$ is the vector of market shares at time $t$ for the initial distribution of market shares $r$ and the sequence of realizations of states $\omega$.

Given stationary portfolio rules $\left(\lambda^{i}\right)$, one is particularly interested in those distribution of wealth shares that evolve in a stationary fashion over time. Here we restrict ourselves to deterministic distributions of market shares, i.e. those that are fixed under the market selection process (17). This restriction might seem to be very strong but actually it is not. Before discussing this point let us specify the notion of a deterministic fixed point. Given portfolio rules $\left(\lambda^{i}\right)$, denote by $\varphi$ the associated random dynamical system.

A state $\bar{r} \in \Delta^{I}$ is called a (deterministic) fixed point of $\varphi$ if, for all $\omega \in \Omega$ and all $t$,

$$
\bar{r}=\varphi(t, \omega, \bar{r}) .
$$

The vector of market shares $\bar{r}$ is also said to be invariant under the market selection process (17).

By the definition of $\varphi(t, \omega, r)$, condition (19) is equivalent to $\bar{r}=\varphi(1, \omega, \bar{r})$ for all $\omega$, i.e. a deterministic state is fixed under the one-step map if and only if it is 
fixed under all $t$-step maps. It is straightforward to see that any state in which one portfolio rule owns the entire wealth does not change over time, i.e. in any set of portfolio rules each unit vector (i.e. each vertex of $\Delta^{I}$ ) is a fixed point. This follows from Proposition 1 which shows that $r^{i}=0$ implies $\varphi^{i}(t, \omega, r)=0$.

To elaborate on the degree of restrictiveness of only considering fixed points, consider a situation in which several portfolio rules co-exist and the distribution of market shares is a stationary process that obeys the law of motion. In other words, there is a random vector $\bar{r}\left(\omega^{t}\right) \in \Delta^{I}$ such that $\bar{r}\left(\omega^{t+1}\right)=\varphi\left(t, \omega, \bar{r}\left(\omega^{t}\right)\right)$ for all $t, \omega$. This is a more general, stochastic fixed point concept, see e.g. SchenkHoppé (2001) for a detailed discussion. One can now define a new portfolio rule $\hat{\lambda}(\omega)=\sum_{i=1}^{I} \lambda^{i}(\omega) \bar{r}^{i}(\omega)$. This portfolio rule inherits stationary, and in any market with $\hat{\lambda}$ managing total wealth, prices are identical to those in the original model. In terms of evolutionary stability, as introduced below, these two models are not exactly equivalent but the latter provides necessary conditions for stability as well as sufficient conditions for instability. This is so because introducing a new portfolio rule reduces the market share of all incumbent rules by the same proportion.

The following result even holds without conditions (B.2)-(B.3).

Proposition 2. Suppose the dividend plus capital gain matrix has full rank at a deterministic fixed point. Then all portfolio rules with positive wealth are identical.

Proof. Equations (7) and (16) give

$$
r_{t+1}^{i}=\sum_{k=1}^{K}\left(\lambda_{0} d_{t+1}^{k}\left(\omega_{t+1}\right)+q_{t+1}^{k}\left(\omega^{t+1}\right)\right) \frac{\lambda_{t, k}^{i}\left(\omega^{t}\right) r_{t}^{i}}{q_{t}^{k}\left(\omega^{t}\right)}
$$

with

$$
q_{t}^{k}\left(\omega^{t}\right)=\sum_{i=1}^{I} \lambda_{t, k}^{i}\left(\omega^{t}\right) r_{t}^{i}
$$

Suppose $r_{t+1}^{i}=r_{t}^{i}=r^{i}>0$ for all $i$. Then equation (20) can be written as

$$
\left(\sum_{k=1}^{K}\left[\lambda_{0} d_{t+1}^{k}\left(\omega_{t+1}\right)+q_{t+1}^{k}\left(\omega^{t+1}\right)\right] \frac{\lambda_{t, k}^{i}\left(\omega^{t}\right)}{q_{t}^{k}\left(\omega^{t}\right)}-1\right) r^{i}=0 .
$$

If the dividend plus capital gain matrix

$$
\lambda_{0} d_{t+1}^{k}\left(\omega_{t+1}\right)+q_{t+1}^{k}\left(\omega^{t+1}\right)
$$

has full rank (as a function of $k$ and $\omega_{t+1}$ for each given history $\omega^{t}$ ), then (22) implies $\lambda_{t, k}^{i}\left(\omega^{t}\right)=q_{t}^{k}\left(\omega^{t}\right)$. In light of (21), this means that for all $i=1, \ldots, I$

$$
\lambda_{t, k}^{i}=\sum_{j=1}^{I} \lambda_{t, k}^{j} r^{j}
$$

Hence $\lambda^{i}$ and $\lambda^{j}$ are identical for all $i, j$. 
In this paper we are only interested in stable fixed points of the market selection process. Loosely speaking, stability means that small perturbations of the initial market share distribution do not have a long-run effect. If a fixed point of market shares is stable, every path of market shares starting in a neighborhood of this fixed point becomes asymptotically identical to it. Since fixed points are associated to unique portfolio rules by Proposition 2 (the total wealth being concentrated on one portfolio rule), the natural definition of a portfolio rule's stability is that of the fixed point's stability. Two different notions of stability will be needed. They are defined as follows.

In what follows the analysis is restricted to the case of two portfolio rules. One pursued by the incumbent and the other by the mutant, or entrant. It is assumed that for any given incumbent portfolio rule $\lambda^{i}$, mutant portfolio rules $\lambda^{j}$ are distinct in the sense that they produce a different payoff stream with certainty. Otherwise it might happen that incumbent and mutant cannot be distinguished by their payoffs. We assume that for almost every $\omega$ there are infinitely many points in time $t$ such that $\lambda^{i}\left(\omega^{t}\right) \neq \lambda^{j}\left(\omega^{t}\right)$. We write $\lambda^{j} \neq \lambda^{i}$ to express this property. ${ }^{3}$ The first entry in the pair of wealth shares $r=\left(r^{i}, r^{j}\right)$ refers to the incumbent, while the other refers to the entrant.

Definition 1. A portfolio rule $\lambda^{i}$ is called evolutionary stable, iffor every portfolio rule $\lambda^{j} \neq \lambda^{i}$ there is a random variable $\varepsilon>0$ such that $\lim _{t \rightarrow \infty} \varphi^{i}(t, \omega, r)=1$ for all $r^{i} \geq 1-\varepsilon(\omega)$ almost surely.

For each evolutionary stable portfolio rule there exits an entry barrier (a random variable here) below which no new portfolio rule gains against the incumbent's rule. Any perturbation of the distribution of market shares, if sufficiently small, does not change the long-run behavior. The market selection process asymptotically leaves the mutant with no market share.

Finally, a corresponding stability criterion for local mutations is introduced.

Definition 2. A portfolio rule $\lambda^{i}$ is called locally evolutionary stable, if there exists a random variable $\delta(\omega)>0$ such that $\lambda^{i}$ is evolutionary stable for all portfolio rules $\lambda^{j} \neq \lambda^{i}$ with $\left\|\lambda^{i}(\omega)-\lambda^{j}(\omega)\right\|<\delta(\omega)$ for all $\omega$.

A locally evolutionary stable portfolio rule is evolutionary stable with respect to local mutations. That is, mutants' portfolio rules are restricted to small deviations from the status quo rule.

Consider again the case in which several portfolio rules co-exist in the market. Evolutionary stability of $\hat{\lambda}(\omega)=\sum_{i=1}^{I} \lambda^{i}(\omega) \bar{r}^{i}(\omega)$ implies that the state $\bar{r}(\omega)$ is stable against local deviations which reduce all incumbents' market shares by the same proportion, i.e. in the perturbation in the original system is perpendicular to the face of the simplex in which $\bar{r}(\omega)$ is located. For instance if there is one mutant, the perturbed system starts at $\left(\varepsilon(\omega),(1-\varepsilon(\omega)) \bar{r}^{1}(\omega), \ldots,(1-\varepsilon(\omega)) \bar{r}^{I}(\omega)\right)$. Thus evolutionary stability (instability) of the fixed point which assigns all wealth to

3 Under ergodic states of the world this set has probability one or zero. This holds in particular if the state is an i.i.d. or irreducible Markov process. Assuming that this property holds with positive probability then ensures that it holds almost surely. 
$\hat{\lambda}(\omega)$ is a necessary (sufficient) condition for evolutionary stability (instability) of the random fixed point $\bar{r}(\omega)$.

\section{The main result}

A detailed analysis is provided of the evolutionary stability of stationary portfolio rules. The local (in)stability conditions obtained here lead to a unique evolutionary stable portfolio rule, provided the relative dividend payoffs are governed by a stationary Markov process. The derivation of these conditions uses an approach that is motivated by the technique first applied for short-lived asset in Hens and Schenk-Hoppé (2005) - though the model in that paper is considerably simpler.

To analyze evolutionary stability of a portfolio rule, one has to consider the random dynamical system (17) with an incumbent (with market share $r_{t}^{1}$ ) and a mutant (with market share $r_{t}^{2}=1-r_{t}^{1}$ ). The resulting one-dimensional system governing the market selection process for two stationary portfolio rules is given by

$$
r_{t+1}^{1}=\frac{\lambda_{0}}{\delta_{t+1}}\left(\left[1-\sum_{k=1}^{K} \lambda_{t+1, k}^{2} \theta_{t, k}^{2}\right] \sum_{k=1}^{K} d_{t+1}^{k} \theta_{t, k}^{1}+\left[\sum_{k=1}^{K} \lambda_{t+1, k}^{2} \theta_{t, k}^{1}\right] \sum_{k=1}^{K} d_{t+1}^{k} \theta_{t, k}^{2}\right)
$$

where $\lambda_{t, k}^{i}=\lambda_{k}^{i}\left(\omega^{t}\right), d_{t+1}^{k}=d^{k}\left(\omega_{t+1}\right)$, and

$$
\begin{aligned}
\delta_{t+1}= & {\left[1-\sum_{k=1}^{K} \lambda_{t+1, k}^{1} \theta_{t, k}^{1}\right]\left[1-\sum_{k=1}^{K} \lambda_{t+1, k}^{2} \theta_{t, k}^{2}\right] } \\
& -\left[\sum_{k=1}^{K} \lambda_{t+1, k}^{2} \theta_{t, k}^{1}\right]\left[\sum_{k=1}^{K} \lambda_{t+1, k}^{1} \theta_{t, k}^{2}\right] .
\end{aligned}
$$

The portfolio of the incumbent and the mutant, respectively, are given by

$$
\theta_{t, k}^{1}=\frac{\lambda_{t, k}^{1} r_{t}^{1}}{\lambda_{t, k}^{1} r_{t}^{1}+\lambda_{t, k}^{2}\left(1-r_{t}^{1}\right)} \quad \text { and } \quad \theta_{t, k}^{2}=\frac{\lambda_{t, k}^{2}\left(1-r_{t}^{1}\right)}{\lambda_{t, k}^{1} r_{t}^{1}+\lambda_{t, k}^{2}\left(1-r_{t}^{1}\right)} .
$$

Denote the right-hand side of (23) by $h\left(\omega^{t+1}, r_{t}^{1}\right)$. The variational equation $v_{t+1}=\left[\partial h\left(\omega^{t+1}, r_{t}^{1}\right) /\left.\partial r_{t}^{1}\right|_{r_{t}^{1}=1}\right] v_{t}$ governs the stochastic dynamics of the linearization of (23) at the fixed point $r_{t}^{1} \equiv 1$. It is derived from the derivative of (23)'s right-hand side with respect to $r_{t}^{1}$ evaluated at $r_{t}^{1}=1$. This derivative can be equated as

$$
\left.\frac{\partial h\left(\omega^{t+1}, r_{t}^{1}\right)}{\partial r_{t}^{1}}\right|_{r_{t}^{1}=1}=\sum_{k=1}^{K}\left(\lambda_{k}^{1}\left(\omega^{t+1}\right)+\lambda_{0} d^{k}\left(\omega_{t+1}\right)\right) \frac{\lambda_{k}^{2}\left(\omega^{t}\right)}{\lambda_{k}^{1}\left(\omega^{t}\right)}
$$

(The necessary calculations are lengthy but elementary and therefore omitted.)

From (24) one can read off the exponential growth rate of portfolio rule $\lambda^{2}$,s market share in a small neighborhood of $r^{1}=1$, i.e. the state in which portfolio rule 
$\lambda^{1}$ owns total market wealth. Before going into detail, let us make an assumption on the process that governs the state of nature and in turn determines the asset payoffs.

Throughout the following the analysis is restricted to the stationary Markov case. It is imposed that

(C) The state of nature follows a Markov process with strictly positive transition probabilities, i.e. $\pi_{s \tilde{s}}>0$ for all $s, \tilde{s} \in S$.

Together with the stationarity assumption, (C) implies that the state of nature is governed by an ergodic process.

The exponential growth rate of portfolio rule $\lambda^{2}$ 's wealth share in a small neighborhood of $r^{1}=1$ is given by the Lyapunov exponent of the above variational equation. It is given by

$$
g_{\lambda^{1}}\left(\lambda^{2}\right)=\int_{S^{N}} \sum_{s \in S} \pi_{\omega_{0} s} \ln \left[\sum_{k=1}^{K}\left(\lambda_{k}^{1}\left(\omega^{0}, s\right)+\lambda_{0} d^{k}(s)\right) \frac{\lambda_{k}^{2}\left(\omega^{0}\right)}{\lambda_{k}^{1}\left(\omega^{0}\right)}\right] \mathbb{P}\left(d \omega^{0}\right)
$$

where $\mathbb{P}$ denotes the stationary probability measure on histories $\omega^{t}$ induced by the Markov chain.

$g_{\lambda^{1}}\left(\lambda^{2}\right)$ can be interpreted as the growth rate of the portfolio rule $\lambda^{2}$ at $\lambda^{1}$-prices because for $r^{1}=1$ the asset prices correspond to the budget shares of portfolio rule $\lambda^{1}$ and thus to the vector $\lambda^{1}$. Clearly asset prices are not constant but change over time due to changes in the wealth allocation of the incumbent's portfolio rule $\lambda^{1}$. For instance at time $t$, prices are equal to the vector $\lambda^{1}\left(\omega^{t}\right)$.

This growth rate determines the local stability of the fixed point $r^{1}=1$, i.e. the qualitative properties of the original, non-linear system's local dynamics is governed by that of its linearization, see Hens and Schenk-Hoppé (2005) for a detailed account. If the growth rate is negative, $g_{\lambda^{1}}\left(\lambda^{2}\right)<0$, portfolio rule $\lambda^{2}$ loses market share while portfolio rule $\lambda^{1}$ 's market share tends to one. In this case the portfolio rule $\lambda^{1}$ is stable against $\lambda^{2}$. If the growth rate is positive, $g_{\lambda^{1}}\left(\lambda^{2}\right)>$ 0 , portfolio rule $\lambda^{2}$ gains market share while that of rule $\lambda^{1}$ falls. In this case the portfolio rule $\lambda^{1}$ is not stable against $\lambda^{2}$. The latter property defines (local) evolutionary instability of a portfolio rule.

Our main result shows that this (in)stability condition can be employed to single out a unique evolutionary stable portfolio rule. Moreover, an explicit representation can be given for this portfolio rule

Theorem 1. Define the portfolio rule $\lambda^{*}$ by $\lambda_{0}^{*}=\lambda_{0}$ and

$$
\lambda^{*}=\lambda_{0} \sum_{m=1}^{\infty}\left(1-\lambda_{0}\right)^{m} \pi^{m} d
$$

using the matrix notation $\lambda^{*}=\left(\lambda_{k}^{*}(s)\right)_{s, k}$ and $d=\left(d^{k}(s)\right)_{s, k}$. 


\section{Stability}

(i) Suppose $\left[\lambda_{k}^{*}(s)+\lambda_{0} d^{k}(s)\right]_{s, k}$ has full rank. Then for every portfolio rule $\lambda \neq \lambda^{*}$, one has $g_{\lambda^{*}}(\lambda)<0$. Thus $\lambda^{*}$ is evolutionary stable.

(ii) For every $\lambda$, one has $g_{\lambda^{*}}(\lambda) \leq 0$. Thus $\lambda^{*}$ is never evolutionary unstable.

\section{Instability}

(iii) For every $\lambda \neq \lambda^{*}$ there exist arbitrarily close portfolio rules $\mu \neq \lambda$ such that $g_{\lambda}(\mu)>0$. Thus every $\lambda \neq \lambda^{*}$ is locally evolutionary unstable and, in particular, evolutionary unstable.

A proof of Theorem 1 is provided at the end of this section.

The portfolio rule $\lambda^{*}$ defined in Theorem 1 is a stationary vector of budget shares and forms a Markov process that only depends on the current state of nature. The portfolio rule $\lambda^{*}$ is well-defined because $\pi^{m} d$ is bounded and $1-\lambda_{0}<1$.

According to the portfolio rule $\lambda^{*}$ one has to divide wealth across assets according to the expected discounted value of their (relative) future dividend payoffs. The discounting rate is given by the saving rate $1-\lambda_{0}$, and the expected value is taken with respect to the objective probability measure. If the $\lambda^{*}$ portfolio rule manages all market wealth then all asset prices are given by this vector of fundamental values. In this respect the $\lambda^{*}$-rule corresponds to a rational market.

Theorem 1 shows that the $\lambda^{*}$-rule has the following properties. A $\lambda^{*}$ market is evolutionary stable, it cannot be invaded by a portfolio rule that is distinct from $\lambda^{*}$. Every non- $\lambda^{*}$ market is not robust against small deviations from the status quo. The stronger version of the first finding requires that every mutant portfolio generates a different payoff stream-this is ensured here by the full rank condition.

The intuition for the main result is immediate from the expression for the exponential growth rate (25). From this equation one can read off that $\lambda^{*}$ is the portfolio rule with the highest exponential growth rate in any population where itself determines market prices. In this sense the $\lambda^{*}$ portfolio rule plays the "best response against itself." (25) also shows that the $\lambda^{*}$ portfolio rule is an equilibrium in a standard two-period economy with a log-utility investor. Therefore the $\lambda^{*}$ portfolio rule yields the maximum growth portfolio and $\lambda^{*}$-prices prevail in such an economy.

The budget shares of the $\lambda^{*}$ portfolio rule have the following remarkable property. From the definition (26) one can easily verify that $\lambda^{*}$ satisfies

$$
\mathbb{E}\left(\lambda_{k}^{*} \mid s\right)+\lambda_{0} \mathbb{E}\left(d^{k} \mid s\right)=\lambda_{k}^{*}(s) /\left(1-\lambda_{0}\right)
$$

for all $k$ and all $s .{ }^{4}$ That is, the $\lambda^{*}$ portfolio rule "balances" capital and dividend gains. Due to the Markov structure of dividends, an adjustment is necessary whenever the conditional expected future payoff $\mathbb{E}\left(d^{k} \mid s\right)$ changes.

Few remarks on the case of i.i.d. dividend payments are in order. If the state of nature is i.i.d., the transition probability matrix has constant rows, each with

\footnotetext{
${ }^{4}$ The conditional expected value is defined as $\mathbb{E}\left(d^{k} \mid s\right)=\sum_{\tilde{s}} \pi_{s \tilde{s}} d^{k}(\tilde{s})$. The term $\left(1-\lambda_{0}\right)$ on the right-hand side appears because $\sum_{k=1}^{K} \lambda_{k}^{*}(s)=1-\lambda_{0}$.
} 
the probability of the corresponding state. The portfolio rule is straightforward to equate as $\lambda_{k}^{*}=\left(1-\lambda_{0}\right) \mathbb{E} d^{k}$, i.e. the budget shares are equal to the assets' expected payoff. Again in a $\lambda^{*}$ market each asset is priced at its fundamental value. Moreover, in the i.i.d. case the budget shares are fixed since the current state of nature does not provide any valuable information on the future dividend payoffs.

Let us finally give a result on the full rank condition in Theorem 1(i).

\section{Lemma 1. The condition}

$$
\left[\lambda_{k}^{*}(s)+\lambda_{0} d^{k}(s)\right]_{s, k} \text { has full rank }
$$

\section{is satisfied}

(i) in the i.i.d. case, if and only if $\left[d^{k}(s)\right]_{s, k}$ has full rank.

(ii) in the Markov case, if and only if $\left[d^{k}(s)\right]_{s, k}$ and $\left[\operatorname{Id}-\left(1-\lambda_{0}\right) \pi\right]$ have full rank.

Proof. In the i.i.d. case, $\lambda_{k}^{*}=\left(1-\lambda_{0}\right) \mathbb{E} d^{k}$, i.e. every column vector of the matrix $\lambda^{*}$ is constant. As all row sums of $d=\left[d^{k}(s)\right]_{s, k}$ are equal to 1 , adding a constant column vector does not change $d$ 's rank. Therefore, if $d$ has full rank then so does $\lambda^{*}+\lambda_{0} d$. And the converse also holds true.

In the Markov case, notice that $\lambda^{*}+\lambda_{0} d=\lambda_{0}\left[\sum_{m=0}^{\infty}\left(1-\lambda_{0}\right)^{m} \pi^{m}\right] d=$ $\lambda_{0}\left[\operatorname{Id}-\left(1-\lambda_{0}\right) \pi\right]^{-1} d$. Assertion (ii) follows straightforwardly from this representation.

The proof also shows that the full rank condition is satisfied if $d$ has full rank and the Markov transition matrix is sufficiently close to a matrix with constant rows (as is true in the i.i.d. case). Let $\pi$ be the transition matrix of an i.i.d. process, and denote all (constant) entries on row $s$ by $p_{s}$. Then Id $-\left(1-\lambda_{0}\right) \pi$ has a columndominant diagonal, if $1-\left(1-\lambda_{0}\right) p_{s}>\sum_{\bar{s} \neq s}\left(1-\lambda_{0}\right) p_{\bar{s}}$. The right-hand side is equal to $\left(1-\lambda_{0}\right)\left(1-p_{s}\right)$. As $\lambda_{0}>0$, the previous inequality holds. Continuity now ensures the above assertion.

We conclude the section by providing the main result of this paper.

Proof of Theorem 1. We prove assertion (iii) before treating (i) and (ii). For notational simplicity, let us normalize portfolio rules with $1-\lambda_{0}$ to achieve $\sum_{k=1}^{K} \lambda_{k}=1$, and let us denote $\lambda=\left(\lambda_{1}, . ., \lambda_{K}\right) \in \Delta^{K}$.

Let $\lambda \neq \lambda^{*}$. To prove assertion (iii), it suffices to show that $g_{\lambda}(\mu)>0$ for some $\mu$ in a given neighborhood of $\lambda$. Using (25), the growth rate of portfolio rule $\mu$ at $\lambda$-prices can be written as

$$
g_{\lambda}(\mu)=\int_{S^{N}} \tilde{g}_{\lambda}\left(\mu\left(\omega^{0}\right), \omega_{0}\right) \mathbb{P}\left(d \omega^{0}\right)
$$

with

$$
\tilde{g}_{\lambda}\left(\mu\left(\omega^{0}\right), \omega_{0}\right)=\sum_{s \in S} \pi_{\omega_{0} s} \ln \left[\sum_{k=1}^{K}\left[\left(1-\lambda_{0}\right) \lambda_{k}\left(\omega^{0}, s\right)+\lambda_{0} d^{k}(s)\right] \frac{\mu_{k}\left(\omega^{0}\right)}{\lambda_{k}\left(\omega^{0}\right)}\right] .
$$

Obviously, $\tilde{g}_{\lambda}\left(\lambda\left(\omega^{0}\right), \omega_{0}\right) \equiv 0$ and, for every fixed $\omega_{0}, \mu \rightarrow \tilde{g}_{\lambda}\left(\mu, \omega_{0}\right)$ is a concave function. 
Assertion (iii) is proved if it can be established that, for every $\lambda \neq \lambda^{*}, g_{\lambda}(\mu)$ does not take on a local maximum at $\lambda=\mu$.

One has

$$
\begin{aligned}
& \frac{\partial \tilde{g}_{\lambda}\left(\mu\left(\omega^{0}\right), \omega_{0}\right)}{\partial \mu_{n}\left(\omega^{0}\right)} \\
& =\sum_{s \in S} \pi_{\omega_{0} s} \frac{\left[\left(1-\lambda_{0}\right) \lambda_{n}\left(\omega^{0}, s\right)+\lambda_{0} d^{n}(s)\right] / \lambda_{n}\left(\omega^{0}\right)}{\sum_{k=1}^{K}\left[\left(1-\lambda_{0}\right) \lambda_{k}\left(\omega^{0}, s\right)+\lambda_{0} d^{k}(s)\right] \mu_{k}\left(\omega^{0}\right) / \lambda_{k}\left(\omega^{0}\right)}
\end{aligned}
$$

Thus

$$
\begin{aligned}
& \sum_{n=1}^{K}\left(\left.\frac{\partial \tilde{g}_{\lambda}\left(\mu\left(\omega^{0}\right), \omega_{0}\right)}{\partial \mu_{n}\left(\omega^{0}\right)}\right|_{\mu\left(\omega^{0}\right)=\lambda\left(\omega^{0}\right)}\right) d \mu_{n}\left(\omega^{0}\right) \\
& =\sum_{n=1}^{K} \frac{\sum_{s \in S} \pi_{\omega_{0} s}\left[\left(1-\lambda_{0}\right) \lambda_{n}\left(\omega^{0}, s\right)+\lambda_{0} d^{n}(s)\right]}{\lambda_{n}\left(\omega^{0}\right)} d \mu_{n}\left(\omega^{0}\right)
\end{aligned}
$$

for every $d \mu_{1}\left(\omega^{0}\right), \ldots, d \mu_{K}\left(\omega^{0}\right)$ with $\sum_{n=1}^{K} d \mu_{n}\left(\omega^{0}\right)=0$.

We now show that (30) is strictly positive for some $\left(d \mu_{1}\left(\omega^{0}\right), \ldots, d \mu_{K}\left(\omega^{0}\right)\right)$ with $\sum_{n=1}^{K} d \mu_{n}\left(\omega^{0}\right)=0$ provided $\lambda\left(\omega^{0}\right) \neq \lambda^{*}\left(\omega_{0}\right)$. This property implies existence of some $\mu\left(\omega^{0}\right)$, arbitrarily close to $\lambda\left(\omega^{0}\right)$, with $\tilde{g}_{\lambda}\left(\mu\left(\omega^{0}\right), \omega_{0}\right)>0$.

We then choose such a $\mu\left(\omega^{0}\right)$ for all $\omega^{0}$ with $\lambda\left(\omega^{0}\right) \neq \lambda^{*}\left(\omega_{0}\right)$ (which happens with some strictly positive probability by the assumption $\left.\lambda \neq \lambda^{*}\right)$ and set $\mu\left(\omega^{0}\right)=$ $\lambda\left(\omega^{0}\right)$ otherwise. This defines a portfolio rule $\mu$ that can be arbitrarily close to $\lambda$. Measurability of $\mu$ follows from the fact that, due to finiteness of $S$, the sigma algebra of the probability space under consideration is the power set (and thus every function is measurable). By construction the portfolio rule $\mu$ satisfies $g_{\lambda}(\mu)>0$, which verifies assertion (iii).

It is clear that (30) is strictly positive for some $\left(d \mu_{1}\left(\omega^{0}\right), \ldots, d \mu_{K}\left(\omega^{0}\right)\right)$ with $\sum_{n=1}^{K} d \mu_{n}\left(\omega^{0}\right)=0$ if and only if

$$
\frac{\sum_{s \in S} \pi_{\omega_{0} s}\left[\left(1-\lambda_{0}\right) \lambda_{n}\left(\omega^{0}, s\right)+\lambda_{0} d^{n}(s)\right]}{\lambda_{n}\left(\omega^{0}\right)}
$$

is not constant in $n$ (for given $\omega^{0}$ ).

We will show that $(31)$ is constant, i.e.

$$
\sum_{s \in S} \pi_{\omega_{0} s}\left[\left(1-\lambda_{0}\right) \lambda_{n}\left(\omega^{0}, s\right)+\lambda_{0} d^{n}(s)\right]=c \lambda_{n}\left(\omega^{0}\right)
$$

for all $n$, if and only if $\lambda=\lambda^{*}$.

Taking the sum over $n$ on both sides of the last equality shows that $c=1$. The condition that (31) is constant therefore becomes

$$
\sum_{s \in S} \pi_{\omega_{0} s}\left[\left(1-\lambda_{0}\right) \lambda_{n}\left(\omega^{0}, s\right)+\lambda_{0} d^{n}(s)\right]=\lambda_{n}\left(\omega^{0}\right)
$$

By definition of $\lambda^{*}$, (32) holds if $\lambda=\lambda^{*}$. To show that (32) implies $\lambda=\lambda^{*}$, we need to consider three distinct cases: (a) $\lambda\left(\omega^{0}\right)$ does not depend on $\omega^{0}$; (b) $\lambda\left(\omega^{0}\right)$ 
depends only on a finite history, i.e. $\lambda\left(\omega^{0}\right)=\lambda\left(\omega_{-T}, \ldots, \omega_{0}\right)$ for some $T \geq 0$; and (c) $\lambda\left(\omega^{0}\right)$ depends on an infinite history.

Case (a): In this case (32) takes the form

$$
\left(1-\lambda_{0}\right) \lambda_{n}+\lambda_{0} \mathbb{E}\left(d^{n} \mid \omega_{0}\right)=\lambda_{n}
$$

which is equivalent to $\mathbb{E}\left(d^{n} \mid \omega_{0}\right)=\lambda_{n}$. This can only hold if the dividend process is an i.i.d. process, otherwise it is a contradiction. But in the i.i.d. case, $\lambda_{n}^{*}=\mathbb{E} d^{n}$.

Case (b): If $\lambda\left(\omega^{0}\right)$ depends only on $\omega_{0}$, then obviously $\lambda=\lambda^{*}$, cf. (27). For a portfolio rule $\lambda\left(\omega^{0}\right)$ that depends on a history of length $T \geq 1$, (32) becomes

$$
\sum_{s \in S} \pi_{\omega_{0} s}\left[\left(1-\lambda_{0}\right) \lambda_{n}\left(\omega_{-T+1}, \ldots, \omega_{0}, s\right)+\lambda_{0} d^{n}(s)\right]=\lambda_{n}\left(\omega_{-T}, \ldots, \omega_{0}\right)
$$

If $\lambda_{n}$ would vary with $\omega_{-T}$, (34) could not hold for all $\omega^{0}$. Thus (34) implies that $\lambda\left(\omega^{0}\right)=\lambda\left(\omega_{-T+1}, \ldots, \omega_{0}\right)$. Repeated application shows that $\lambda\left(\omega^{0}\right)=\lambda\left(\omega_{0}\right)$. However, this implies $\lambda=\lambda^{*}$, as discussed above.

Case (c): In this case, (32) reads

$$
\sum_{\omega_{1} \in S} \pi_{\omega_{0} \omega_{1}}\left[\left(1-\lambda_{0}\right) \lambda_{n}\left(\omega^{1}\right)+\lambda_{0} d^{n}\left(\omega_{1}\right)\right]=\lambda_{n}\left(\omega^{0}\right) .
$$

An analogous equation holds with $\lambda_{n}\left(\omega^{1}\right)$ on the right-hand side,

$$
\sum_{\omega_{2} \in S} \pi_{\omega_{1} \omega_{2}}\left[\left(1-\lambda_{0}\right) \lambda_{n}\left(\omega^{2}\right)+\lambda_{0} d^{n}\left(\omega_{2}\right)\right]=\lambda_{n}\left(\omega^{1}\right) .
$$

Inserting (36) in (35) yields

$$
\begin{aligned}
\lambda_{n}\left(\omega^{0}\right)= & \left(1-\lambda_{0}\right)^{2} \pi_{\omega_{0} \omega_{2}}^{2} \lambda_{n}\left(\omega^{2}\right) \\
& +\lambda_{0}\left[\left(1-\lambda_{0}\right) \sum_{\omega_{2}} \pi_{\omega_{0} \omega_{2}}^{2} d^{n}\left(\omega_{2}\right)+\sum_{\omega_{1}} \pi_{\omega_{0} \omega_{1}}^{1} d^{n}\left(\omega_{1}\right)\right]
\end{aligned}
$$

where $\pi_{\omega_{0} \omega_{m}}^{m}=\sum_{\omega_{1}, \ldots, \omega_{m}} \pi_{\omega_{0} \omega_{1}} \ldots \pi_{\omega_{m-1} \omega_{m}}$.

Repeating this procedure and observing that

$$
\left(1-\lambda_{0}\right)^{m} \sum_{\omega_{m}} \pi_{\omega_{0} \omega_{m}}^{m} \lambda_{n}\left(\omega^{m}\right) \rightarrow 0 \text { as } m \rightarrow \infty
$$

we find

$$
\lambda_{n}\left(\omega^{0}\right)=\frac{\lambda_{0}}{1-\lambda_{0}} \sum_{m=1}^{\infty}\left(1-\lambda_{0}\right)^{m} \sum_{\omega_{m}} \pi_{\omega_{0} \omega_{m}}^{m} d^{n}\left(\omega_{m}\right)
$$

Thus $\lambda_{n}\left(\omega^{0}\right)$ is a function of $\omega_{0}$ only, implying that $\lambda=\lambda^{*}$, as discussed in case (b).

Assertion (i) can be proved as follows. If the full rank condition holds for some $\omega_{0}$, then $\mu \rightarrow \tilde{g}_{\lambda}\left(\mu, \omega_{0}\right)$ is a strictly concave function. This is so because the full rank assumption in (i) ensures that $\left(\left[\left(1-\lambda_{0}\right) \lambda_{k}^{*}(s)+\lambda_{0} d^{k}(s)\right] / \lambda_{k}^{*}\left(\omega_{0}\right)\right)_{s, k}$ also has full rank. (After the above normalization of portfolio rules, the condition in (i) 
says that $\left(\left(1-\lambda_{0}\right) \lambda_{k}^{*}(s)+\lambda_{0} d^{k}(s)\right)_{s, k}$ has full rank. The above term is obtained by multiplying every column with a strictly positive constant. This operation preserves the full rank.)

Statement (i) is thus obtained if it can be shown that the maximum of $\mu \rightarrow$ $\tilde{g}_{\lambda^{*}}\left(\mu, \omega_{0}\right)$ with $\mu \in \Delta^{K}$ is equal to zero and that this maximum is attained at $\mu\left(\omega^{0}\right)=\lambda^{*}\left(\omega_{0}\right)$. Obviously, $\tilde{g}_{\lambda^{*}}\left(\lambda^{*}\left(\omega_{0}\right), \omega_{0}\right) \equiv 0$. The function $\mu \rightarrow \tilde{g}_{\lambda^{*}}\left(\mu, \omega_{0}\right)$ takes on its maximum at $\mu\left(\omega^{0}\right)=\lambda^{*}\left(\omega_{0}\right)$ if (31) is constant for $\lambda\left(\omega^{0}\right)=\lambda^{*}\left(\omega_{0}\right)$. But this has already been proved.

Assertion (ii) is obvious from the proof of (i). If the full rank condition does not hold, then zero is still the maximum of $\mu \rightarrow \tilde{g}_{\lambda^{*}}\left(\mu, \omega_{0}\right)$. However, the portfolio rule at which this maximum is attained is not necessarily unique. Thus the assertion is weaker than in (i).

\section{Conclusion and outlook}

We have studied the evolution of wealth shares of portfolio rules in arbitrary (complete or incomplete) markets with long-lived assets. Prices are determined endogenously. The performance of a portfolio rule in the process of repeated reinvestment of wealth is determined by the wealth share eventually conquered in competition with other portfolio rules. Using random dynamical systems theory, we derived necessary and sufficient conditions for the evolutionary stability of portfolio rules. In the case of Markov (in particular i.i.d.) payoffs these local stability conditions lead to a (surprisingly) simple portfolio rule that is the only evolutionary stable one. This rule possesses an explicit representation as it invests proportionally to the expected relative dividends. This stability property may help to explain why on long-term averages stock markets look quite rational while severe departures are possible in the short- and medium-term.

As in many other papers on economic theory, our results are based on several assumptions and modeling choices that shall be extended in future research. For example, we have restricted portfolio rules to be adapted to the information filtration given by the revelation of the states of the world that are exogenous to the model. Hence, we did not allow for price dependent portfolio rules as for example simple momentum strategies like "buy (sell) when prices have gone up (down)." Moreover, we made a clear distinction between the market selection process and the mutation process. The latter act at the selection process only once the former has settled at a point of rest. It would be desirable to consider a selection process with ongoing mutations. Finally, in our model the wealth shares of the portfolio rules increase due to "internal growth," i.e. they increase by the returns they have generated. This process shall be augmented by a process of "external growth" in which portfolio rules increase their wealth share by attracting wealth from less successful rules. Data from Mutual Funds and Hedge Funds, for example, show that internal growth leads external growth so that one effect of this extension my be speeding up the market selection process. However, this and the other possible extensions mentioned have to be checked carefully in future research. 


\section{References}

Algoet, P.H., Cover, T.M.: Asymptotic optimality and asymptotic equipartition properties of logoptimum investment. Annals of Probability 16, 876-898 (1988)

Arnold, L.: Random dynamical systems. Berlin Heidelberg New York: Springer 1998

Blume, L., Easley, D.: If you are so smart why aren't you rich? Belief selection in complete and incomplete markets. Mimeo, Cornell University (2001)

Breiman, L.: Optimal gambling systems for favorable games. Fourth Berkeley Symposium on Mathematical Statistics and Probability 1, 65-78 (1961)

Cover, T.M.: An algorithm for maximizing expected log-investment return. IEEE Transformation Information Theory 30, 369-373 (1984)

Cover, T.M.: Universal portfolios. Mathematical Finance 1, 1-29 (1991)

De Long, J.B., Shleifer, A., Summers, L.H., Waldmann, R.J.: Noise trader risk in financial markets. Journal of Political Economy 98, 703-738 (1990)

Evstigneev, I.V., Hens, T., Schenk-Hoppé, K.R.: Market selection of financial trading strategies: global stability. Mathematical Finance 12, 329-339 (2002)

Fama,E.: The behavior of stock market prices. Journal of Business 38, 34-105 (1965)

Friedman, M.: Essays in positive economics. Chicago: University of Chicago Press 1953

Hakansson, N.: Optimal investment and consumption strategies under risk for a class of utility functions. Econometrica 38, 587-607 (1970)

Hens, T., Schenk-Hoppé, K.R.: Evolutionary stability of portfolio rules in incomplete markets. Journal of Mathematical Economics 41, 43-66 (2005)

Hens, T., Schenk-Hoppé, K.R., Stalder, M.: An application of evolutionary finance to firms listed in the Swiss market index. Swiss Journal of Economics and Statistics 138, 465-487 (2002)

Karatzas, I., Shreve, S.E.: Methods of mathematical finance. Berlin Heidelberg New York: Springer 1998

Kelly, J.L.: A new interpretation of information rate. Bell System Technical Journal 35, 917-926 (1956)

Kraus, A., Litzenberger, R.H.: Market equilibrium in a multiperiod state preference model with logarithmic utility. Journal of Finance 30, 1213-1227 (1975)

Lucas, R.: Asset prices in an exchange economy. Econometrica 46, 1429-1445 (1978)

Luenberger, D.G.: Investment science. New York: Oxford University Press 1997

Murata, Y.: Mathematics for stability and optimization of economic systems. New York: Academic Press 1977

Sandroni, A.: Do markets favor agents able to make accurate predictions? Econometrica 68, 1303-1341 (2000)

Schenk-Hoppé, K.R.: Random dynamical systems in economics. Stochastics and Dynamics 1, 63-83 (2001)

Shiller, R.J.: Do stock prices move too much to be justified by subsequent changes in dividends? American Economic Review 71, 421-436 (1981)

Thorp, E.O.: Portfolio choice and the Kelly criterion. In: Ziemba, W.T., Vickson, R.G. (eds.) Stochastic models in finance, pp. 599-619. New York: Academic Press 1971 